\title{
Impact of Discordance Among Regulations for Biopharmaceutics Classification System-Based Waivers of Clinical Bioequivalence Studies
}

\author{
Jack Cook \\ Pfizer Inc., 445 Eastern Point Road, MS8260-2504, Groton, CT 06340
}

\begin{abstract}
Approximately 10 years ago, the FDA issued a guidance whereby dissolution could be used to establish bioequivalence for highly soluble and highly permeable immediate-release products. This pathway has advantages in lower costs for pharmaceutical companies and avoids unnecessary exposure of human subjects who would derive no medical benefit from participating in bioequivalence studies. Subsequently, other agencies have also adopted similar guidances. However, there are differences among agency guidances that significantly impact the number of compounds eligible for BCS-based biowaivers across all regulatory agencies. These include differences in BCS Classes for which biowaivers are considered, differences in the definition of highly permeable, and differences in the definition of highly soluble. The currently low rate of biowaiver submissions may in part be due to these differences.
\end{abstract}

KEYWORDS: Dissolution; BCS; biowaivers; bioequivalence; permeability.

\section{INTRODUCTION}

A midon and coworkers (1) introduced the biopharmaceutic classification system (BCS) in 1995. The paper asserted that by recognizing that drug dissolution and intestinal permeability were the principle processes controlling the rate and extent of absorption, one could develop a scheme to predict the expected correlation between in vitro dissolution and in vivo bioavailability. In 2005, the FDA published the first regulatory guidance (2) that provided a BCS-based pathway for demonstration of bioequivalence through dissolution. It has been estimated (3) that between 555 and 639 bioequivalence studies are run each year, and with a typical study cost of US\$320,000, there is the potential for significant savings through application of the FDA guidance.

Subsequent to the issuance of the FDA guidance, a number of other agencies have issued their own guidances. These include the EMA (4) as well as Health Canada, Australia, ASEAN, China, South Korea, Switzerland, and the WHO (5). These subsequent guidances differ from the FDA guidance in some of their requirements regarding which drug products are eligible for BCS-based waivers of in vivo studies (biowaiver). These differences reduce the number of products that are eligible for biowaivers in all countries.

For innovator companies, bioequivalence trials typically compare a new formulation to their currently marketed formulation, which is used as the reference. Since the marketed formulation is typically the same worldwide, the data package to demonstrate bioequivalence can be the same regardless of where it is to be submitted. To do otherwise would involve doing clinical bioequivalence studies to support some submissions and BCS characterization and dissolution studies to support others. Such a strategy would unnecessarily drive up development costs.

There are a number of advantages for demonstrating bioequivalence through dissolution via a biowaiver relative to a clinical study. These include significant reduction in time and cost as well as avoiding unnecessary exposure of healthy subjects to drugs from which they will receive no medical benefit (3). However, because of differences in regulatory requirements among countries, a drug may qualify for a biowaiver in some countries but not be eligible in others. In these cases, innovator companies will tend to default to human bioequivalence studies as they are universally acceptable. The purpose of this paper is to estimate how much these differences reduce the pool of eligible compounds that will universally qualify for biowaivers. In addition, the author will comment on ways in which regulatory agencies may start to consider harmonization.

\section{METHODS}

To investigate the impact of the discordance among regulations for biowaivers, differences between the United States (2) and EMA (4) guidances were considered as it was felt that the comparison would highlight the most impactful differences. Other agencies have aligned their guidances to be identical to the United States or EMA requirements, and thus the differences are not the result of a single agency being different from all others. The differences considered were:

- BCS Classes for which biowaivers are considered

- Definition of highly permeable.

- Definition of highly soluble. 
Studies from the literature were used to assess the largest set of potential compounds that might be eligible for biowaivers as well as the impact of the differences between the guidances in terms of the reduction in the percentage of possibly eligible compounds where biowaivers could be considered.

\section{RESULTS}

\section{Potential Maximum Pool of Eligible Compounds}

The largest set of potential compounds would consider both BCS Class 1 (highly soluble with high permeability) and Class 3 (highly soluble with low permeability) compounds and would utilize the least restrictive definitions among guidances for highly soluble and highly permeable. Takagi and coworkers (6) used such definitions when they determined provisional BCS classification for the top 200 drugs in several markets on this basis. Lindenberg et al. (7) and Kasmin et al. (8) did similar analyses for drugs on the World Health Organization Model List of Essential Medicines, Core List. The results of these two analyses are presented in Table 1.

Table 1. Provisional BCS Classification for Various Groupings of Drugs

\begin{tabular}{|l|ccccc|}
\hline \multirow{2}{*}{\multicolumn{1}{|c|}{ Grouping }} & \multicolumn{5}{c|}{ Percentage of Drugs by BCS Class } \\
\cline { 2 - 6 } & Class I & Class II & Class III & Class IV \\
\hline Top 200 in United States $^{a}$ & 34 & 33 & 26 & 7 \\
Top 200 in Great Britain $^{a}$ & 33 & 33 & 27 & 7 \\
Top 200 in Spain $^{a}$ & 32 & 27 & 30 & 5 \\
Top 200 in Japan $^{a}$ & 36 & 36 & 20 & 7 \\
WHO Essential Medicines Core List $^{b}$ & 31 & 21 & 38 & 11 \\
WHO Essential Medicines Core List $^{c}$ & 36 & 17 & 38 & 9 \\
\hline Median & 34 & 30 & 29 & 7 \\
\hline
\end{tabular}

Note: Within a grouping, only oral immediate-release products considered and percentages calculated from drugs where information was sufficient to allow at least a provisional BCS classification.

${ }^{a}$ Classification based on Dose Number using $250 \mathrm{~mL}$ volume and CLogP method from ref 6 .

${ }^{b}$ Classification using certain and provisional classifications from ref 7 .

'Classification based on Dose Number and CLogP method from ref 8.

Using median values as an estimate across the different groupings and assuming that these numbers are reflective of all marketed compounds, $63 \%$ of all oral immediate-release drug products could be considered for biowaivers (34\% Class 1 plus $29 \%$ Class 3 ). The $63 \%$ is the maximum potential pool and does not consider other biowaiver criteria such as requirements that the drug not be considered to have a narrow therapeutic index or that the product does not include excipients that alter rate or extent of absorption.

It should also be noted that the World Health Organization allows biowaivers for Class 2 (low solubility, high permeability) compounds that are weak acids (9). Weak acids have high solubility in the intestinal $\mathrm{pH}$ range, and thus rapidly dissolving products behave like solutions in this environment. However, evaluations similar to references 6-8 have not been done to estimate the number of compounds in this subclass of BCS Class 2. Thus the impact of this subclass was not evaluated.

\section{BCS Classes for Which Biowaivers Are Considered}

The exclusion of Class 3 compounds from biowaiver candidates reduces the potential pool by $46 \%$ (from $63 \%$ to $34 \%$ of all drugs, see Table 1). An extension of biowaivers to Class 3 compounds was one of the first changes to the original guidance (10). These compounds are highly soluble across the entire physiologically relevant $\mathrm{pH}$ range encountered in the gastrointestinal tract. Investigators have used simulation to demonstrate that by assuring rapid dissolution (i.e., $>85 \%$ dissolved in $15 \mathrm{~min}$ ), one can assure bioequivalence of Class 3 products $(11,12)$. The EMA, Canada, and WHO have all adopted policies that allow biowaivers for Class 3 compounds that meet very rapid dissolution requirements. While the FDA has not yet approved such a policy, it is actively conducting research in this area (e.g., ClinicalTrial.gov, study identifier CT01010698) and has issued a revised draft guidance allowing biowaivers for Class 3 compounds (13).

\section{Definition of Highly Permeable}

The latest EMA guidance (4) indicates that high permeability "should be justified based on reliable investigations in humans," while the FDA guidance (2) allows human studies, animal studies, or in vitro methods to be used. Dr. Mehta (14) reviewed 45 compounds that underwent FDA review for BCS classification; the type of data included is listed in Table 2. There were at most 27 submissions that contained human data (this assumes that no submission contained both human mass balance and absolute bioavailability studies). Under the assumption that this sample is reflective of the type of data available for BCS Class 1 compounds, this would mean that the human-only requirement would preclude $40 \%$ that would otherwise meet BCS Class 1 criteria. Given that high permeability means that drugs not only easily pass through the intestinal membrane but will also likely cross other membranes as well and thus will be exposed to metabolizing enzymes in the intestine and liver, Class I compounds typically undergo extensive metabolism (15). Considering that a number of these compounds may undergo first-pass metabolism, it is not surprising that one might encounter difficulty in meeting permeability criteria with data from human absolute bioavailability or mass-balance studies.

Table 2. Types of Data Used in Permeability Determinations

\begin{tabular}{|l|c|}
\hline \multicolumn{1}{|c|}{ Data Source } & Number of Submissions Containing \\
\hline Human Absolute Bioavailability Study & 11 \\
\hline Human Mass Balance Study & 16 \\
\hline Animal Radiolabeled Drug Study & 5 \\
\hline In Vitro Caco-2 Cell Line Study & 14 \\
\hline Rat Intestinal Perfusion Study & 1 \\
\hline
\end{tabular}

Forty-five submissions reviewed; some submissions may have contained more than one data source. 
The reason for the EMA position of accepting only human data is not clear, so it is difficult to offer possible pathways to harmonization. In vitro models have proved very predictive of human permeability (16). However, it is recognized that these models may not be reflective of certain phenomena observed in humans. The FDA guidance has taken this into account by requiring additional data to ensure that the data can be translated into humans; the requirements include validation of the in vitro or animal methodology through the use of model compounds (compounds with known human permeability characteristics) to establish that the in vitro or animal model gives similar rank-order results, adequately characterizes absorption mechanisms to establish that passive transport is the dominant absorption pathway, and demonstrates stability of the compound in the in vitro model (4). Once the objections to non-human data are known, additional data requirements could be established to allow use of these data.

\section{Definition of Highly Soluble}

The FDA notes that "a drug substance is considered highly soluble when the highest dose strength is soluble in $250 \mathrm{~mL}$ or less of aqueous media over the $\mathrm{pH}$ range of 1-7.5" (2), while the EMA changed from highest dose to a definition stating that a "drug substance is considered highly soluble if the highest single dose administered as immediate-release formulation(s) is completely dissolved in $250 \mathrm{~mL}$ of buffers within the range of $\mathrm{pH} 1-6.8$ at $37 \pm$ $1{ }^{\circ} \mathrm{C}$ " in their most recent guidance (4). Thus the FDA focuses on the highest formulation strength, while the EMA now focuses on the highest labeled dose, which is often two or more times higher (17). Sediq and colleagues (17) examined the effect of changing from highest formulation strength to highest dose on all drugs for which a biowaiver monograph had been published up to June 2011. They found that the classification of 4 of the 27 compounds (15\%) would change from being highly soluble to having low solubility.

For compounds that do not meet the EMA definition of highly soluble, a human bioequivalence trial would need to be conducted. The EMA guidance (4) states, "The bioequivalence study should in general be conducted at the highest strength." Thus for compounds that have changed BCS Class because of the change in solubility criterion from highest strength to highest dose, the guidance advocates testing them under a condition where the dose used would be the highly soluble criterion. It seems incongruous to require testing at a dose where it is recognized that dissolution would allow adequate proof of bioequivalence. Furthermore, considering that the healthy subjects who participate in human bioequivalence trials receive no direct benefit from taking the compound, it is prudent to avoid unnecessary human testing, no matter how low the risk, if there is another reliable method that does not require human drug exposure.
One can also consider the criterion from a risk standpoint and examine the risk that formulation-related differences would only be revealed at doses higher than the highest strength. If these risks are sufficiently high to require a highly soluble criterion to consider the highest labeled dose, human bioequivalence studies should require that the highest labeled dose be studied. Furthermore, it would seem that the risk would be similar or greater for all low solubility compounds, not just those near the solubility limit. However, most if not all regulatory bioequivalence guidances require that the highest strength (or lower) be used in human bioequivalence studies rather than the highest labeled dose. Based on these standard practices and past bioequivalence history, it is suggested that the risk of formulation differences that only appear at doses above the highest strength is sufficiently low to allow the high solubility criterion to be based on the highest strength.

\section{Impact of Regulatory Differences on Drug Development}

One way to judge the impact of the differences among regulatory guidances is to consider the impact on innovator bioequivalence studies. As noted earlier, for innovator companies, the same two formulations, namely the old and new innovator formulations, are compared for a given drug regardless of where the data is to be submitted. Thus the data package is created such that it can be submitted to multiple agencies. In contrast, for a generic company, the reference compound may change depending on where approval is sought.

It has been estimated that innovator pharmaceutical companies perform a combined total of between 84 and 167 bioequivalence studies a year, and a typical study employs 32 subjects and costs US\$320,000 (3). Based on the data from Table 3, the potential maximum number of savings would be a reduction of 53 to 105 studies, which would translate into approximately 1700 to 3400 fewer subjects exposed to a drug and an approximate savings of US\$17 million to US\$34 million. Current differences among regulatory guidances reduce these totals by $73 \%$ (Table 3 ; $[1$ - Total Cumulative Reduction/Base $]=1-17 / 63=73 \%$ ) to $14-28$ studies, approximately $450-900$ subjects, and US\$4.5 million to US\$9 million. The rather small number of studies projected to be saved when considering differences in guidances is somewhat aligned with the rather low number (approximately 2/year) of BCS-based biowaiver requests submitted by innovator companies and reviewed by the FDA (17). While these numbers are not meant to establish causality, they represent a possible reason for the overall small number of biowaiver requests received by regulatory agencies each year.

The encouraging news is that regulatory agencies are seeking harmonization. As mentioned previously, the FDA is considering a pathway to allow biowaivers for Class 3 compounds. Furthermore, regulators, industry, and academia are meeting to find ways to harmonize guidances (e.g., the European Federation for Pharmaceutical 
Table 3. Impact of Differences in BCS-Based Biowaiver Guidances

\begin{tabular}{|l|c|c|}
\hline \multicolumn{1}{|c|}{ Difference } & Reduction (\%) & Candidate Pool (\% of all drugs) $^{\boldsymbol{a}^{\mathbf{a}}}$ \\
\hline Base & NA & 63 \\
\hline $\begin{array}{l}\text { BCS Classes for which biowaivers are considered } \\
\text { (not allowing biowaivers for Class III compounds) }\end{array}$ & 46 & 24 \\
\hline $\begin{array}{l}\text { Definition of highly permeable } \\
\text { (not allowing animal or cell derived data to be primary) }\end{array}$ & 40 & 20 \\
\hline $\begin{array}{l}\text { Definition of highly soluble } \\
\text { (basing criteria on highest dose rather than strength) }\end{array}$ & 15 & 17 \\
\hline
\end{tabular}

Differences are ordered from highest to lowest impact.

${ }^{a}$ Candidate pool values take into consideration values from previous rows (thus are cumulative).

Sciences began its Global Bioequivalence Harmonization Initiative with an international conference held in March 2015 in Amsterdam, The Netherlands). It is hoped that by recognizing the impact and other considerations noted within this manuscript, this harmonization maybe facilitated.

\section{CONCLUSIONS}

Differences among agencies in their BCS-based biowaiver guidances include differences in BCS classes for which biowaivers are considered, in the definition of highly permeable, and in the definition of highly soluble. These differences significantly impact the number of compounds eligible for BCS-based biowaivers across regulatory agencies. The currently low rate of biowaiver submissions may in part be due to these differences.

\section{REFERENCES}

1. Amidon, G. L.; Lennernäs, H.; Shah, V. P.; Crison, J. R. A Theoretical Basis for a Biopharmaceutic Drug Classification: The Correlation of in Vitro Drug Product Dissolution and in Vivo Bioavailability. Pharm. Res. 1995, 12 (3), 413-420. DOI: 10.1023/A:1016212804288.

2. Waiver of In Vivo Bioavailability and Bioequivalence Studies for Immediate-Release Solid Oral Dosage Forms Based on a Biopharmaceutics Classification System; Guidance for Industry; U.S. Department of Health and Human Services, Food and Drug Administration, Center for Drug Evaluation and Research (CDER), U.S. Government Printing Office: Washington, DC, 2000.

3. Cook, J. A.; Davit, B. M.; Polli, J. E. Impact of Biopharmaceutics Classification System-Based Biowaivers. Mol. Pharmaceutics 2010, 7 (5), 1539-1544. DOI: $10.1021 / \mathrm{mp} 1001747$.

4. Guideline on the Investigation of Bioequivalence. CPMP/ EWP/QWP/1401/98 Rev. 1/ Corr; Committee for Medicinal Products for Human Use (CHMP), European Medicines Agency: London, 2010. http://www.ema. europa.eu/docs/en_GB/document_library/Scientific guideline/2010/01/WC500070039.pdf (accessed April
6, 2015).

5. Davit, B.;Braddy, A.C.;Conner, D. P.;Yu, L.X.International Guidelines for Bioequivalence of Systemically Available Orally Administered Generic Drug Products: A Survey of Similarities and Differences. AAPS J. 2013, 15 (4), 974-990. DOI: 10.1208/s12248-013-9499-x.

6. Takagi, T.; Ramachandran, C.; Bermejo, M.; Yamashita, S.; Yu, L. X.; Amidon, G. L. A Provisional Biopharmaceutical Classification of the Top 200 Oral Drug Products in the United States, Great Britain, Spain, and Japan. Mol. Pharmaceutics 2006, 3 (6), 631-643. DOI: 10.1021/ mp0600182.

7. Lindenberg, M.; Kopp, S.; Dressman, J. B. Classification of orally administered drugs on the World Health Organization Model list of Essential Medicines according to the biopharmaceutics classification system. Eur. J. Pharm. Biopharm. 2004, 58 (2), 265-278. DOI: 10.1016/J.EJPB.2004.03.001.

8. Kasim, N. A.; Whitehouse, M.; Ramachandran, C.; Bermejo, M.; Lennernäs, H.; Hussain, A. S.; Junginger, $\mathrm{H}$. E.; Stavchansky, S. A.; Midha, K. K.; Shah, V. P.; Amidon, G. L. Molecular Properties of WHO Essential Drugs and Provisional Biopharmaceutical Classification. Mol. Pharmaceutics 2004, 1 (1), 85-96. DOI: 10.1021/ mp034006h.

9. WHO Expert Committee on Specifications for Pharmaceutical Preparations. Proposal to waive in vivo bioequivalence requirements for WHO Model List of Essential Medicines immediate-release, solid oral dosage forms; WHO Technical Report Series, No. 937, Annex 8; World Health Organization: Geneva, Switzerland, 2006. http://apps.who.int/prequal/info_general/ documents/TRS937/WHO_TRS_937__annex8_eng. pdf (accessed April 6, 2015).

10. Blume, H. H.; Schug, B. S. The biopharmaceutics classification system (BCS): Class III drugs-better candidates for BA/BE waiver? Eur. J. Pharm. Sci. 1999, 9 (2), 117-121. DOI: 10.1016/S0928-0987(99)00076-7.

11. Tsume, Y.; Amidon, G. L. The Biowaiver Extension for BCS Class III Drugs: The Effect of Dissolution Rate 
on the Bioequivalence of BCS Class III ImmediateRelease Drugs Predicted by Computer Simulation. Mol. Pharmaceutics 2010, 7 (4), 1235-1243. DOI: 10.1021/ mp100053q.

12. Yu, L. X.; Ellison, C. D.; Conner, D. P.; Lesko, L. J.; Hussain, A. S. Influence of Drug Release Properties of Conventional Solid Dosage Forms on the Systemic Exposure of Highly Soluble Drugs. AAPS J. 2001, 3 (3), 86-92. DOI: 10.1208/PS030324.

13. Waiver of In Vivo Bioavailability and Bioequivalence Studies for Immediate-Release Solid Oral Dosage Forms Based on a Biopharmaceutics Classification System; Guidance for Industry; U.S. Department of Health and Human Services, Food and Drug Administration, Center for Drug Evaluation and Research (CDER), U.S. Government Printing Office: Washington, DC, May 2015. http://www.fda.gov/downloads/Drugs/ GuidanceComplianceRegulatorylnformation/ Guidances/UCM070246.pdf (accessed May 26, 2015).

14. Mehta, M. Application of Biopharmaceutical Classification System (BCS) in Regulatory Submissions. Presented at the AAPS Webinar [Online], September
9, 2010. http://www2.aaps.org/uploadedFiles/ Content/Sections_and_Groups/Focus_Groups/In_ Vitro_Release_and_Dissolution_Testing/Resources/ IVRDTFGMehta2010.pdf (accessed April 6, 2015).

15. Chen, M.-L.; Amidon, G. L.; Benet, L. Z.; Lennernas, H.; Yu, L. X. The BCS, BDDCS, and Regulatory Guidances. Pharm. Res. 2011, 28 (7), 1774-1778. DOI: 10.1007/ s11095-011-0438-1.

16. Sun, D.; Yu, L. X.; Hussain, M. A, Wall, D. A.; Smith, R. L.; Amidon, G. L. In vitro testing of drug absorption for drug 'developability' assessment: forming an interface between in vitro preclinical data and clinical outcome. Curr. Opin. Drug Discovery Dev. 2004, 7 (1), 75-85. DOI: 10.1007/S11095-011-0438-1.

17. Sediq, A.; Kubbinga, M.; Langguth, P.; Dressman, J. The Impact of the EMA Change in Definition of "Dose" on the BCS Dose-Solubility Ratio: A Review of the Biowaiver Monographs. J. Pharm. Sci. 2014, 103 (1), 65-70. DOI: 10.1002/JPS.23769. 